\title{
Argon branching ratios for spectral intensity calibration: a reply
}

\author{
K. Danzmann* and M. Kock \\ Institut für Plasmaphysik, Universität Hannover, Callinstrasse 38, 3000 Hannover 1, West Germany
}

Received June 1, 1982

Branching ratios have been obtained for 33 lines originating from nine levels in Ar II by Fourier spectroscopic measurements on a high-current hollow cathode. The values are compared with recent literature data.

\section{INTRODUCTION}

In a recent paper, Adams and Whaling ${ }^{1}$ proposed the use of several families of Ar I and Ar II branching ratios for in situ calibration of the relative spectrometer efficiency. They invited other laboratories engaged in spectrophotometry to provide measurements of these branching ratios with the aim of establishing them as secondary intensity standards. We report such measurements for most of the Ar II lines investigated by the above authors.

\section{EXPERIMENTAL METHOD}

Relative line intensities were measured on five spectra recorded with the 1-m Fourier transform spectrometer (FTS) at Kitt Peak National Observatory. ${ }^{2}$ The light source used was a high-current hollow cathode described by Danzmann and Kock. ${ }^{3}$ It was operated in Ar at a pressure of $0.6 \mathrm{mbar}$. The cathode had a length of $100 \mathrm{~mm}$ and a bore of $8.5 \mathrm{~mm}$. Two of the spectra were recorded with an iron cathode at discharge currents of 0.5 and $2.6 \mathrm{~A}$. One spectrum was obtained with a molybdenum cathode at a discharge current of 2.0 A. These spectra covered the $34000-18000-\mathrm{cm}^{-1}$ (2800-5500- $\AA$ ) range. The remaining two spectra covered the $31000-87000-\mathrm{cm}^{-1}(3500-11500-\AA)$ range. They were recorded with an iron cathode at discharge currents of 0.5 and $2.6 \mathrm{~A}$.

The theoretical wave-number resolution was set to 0.04 $\mathrm{cm}^{-1}$ for all runs. No apodization procedure had been applied during the Fourier transformation. The observed minimum linewidth (FWHM) was $0.05 \mathrm{~cm}^{-1}$ at $8700 \mathrm{~cm}^{-1}$.

At about $34000 \mathrm{~cm}^{-1}$, we observed linewidths of 0.13 and $0.18 \mathrm{~cm}^{-1}$ at discharge currents of 0.5 and $2.6 \mathrm{~A}$, respectively.

The relative detection efficiency was calibrated by the branching-ratio method. We compared Fe I branching ratios measured by Adams and Whaling ${ }^{4}$ or computed from $f$ values measured by Blackwell et al. ${ }^{5-8}$ with the intensities of the corresponding lines on the FTS spectra. Additionally, we obtained independent calibration curves for the spectra from the response of the FTS to a tungsten-ribbon filament lamp. In the long-wavelength region $(>4000 \AA)$, the agreement between both calibration procedures was within $2 \%$.

In the short-wavelength region, we used only the response to the tungsten lamp for an interpolation of the calibration curve between the discrete Fe I wavelengths.
There are three main sources for systematic errors in this type of measurement:

1. The lines under investigation may be disturbed by blends, especially by lines belonging to the strong emission spectrum of the material sputtered off the cathode;

2. The intensities of the lines may be falsified by selfabsorption; and

3. Misidentification of incidental coincidence of different lines may occur.

The use of two different cathode materials in our investigation permits an independent check to establish that none of the Ar II lines of interest is disturbed by blends with metal lines.

We used three different discharge currents to check for the effects of self-absorption. It is only for an optically thin light source that the measured branching ratios will be independent of the discharge current.

The high resolution of the FTS in conjunction with the precise wavelength scale for the whole spectrum increases the sensitivity for a correct identification.

\section{RESULTS AND DISCUSSION}

The complete set of branching ratios comprising 33 lines is listed in Table 1, together with the data of Adams and Whaling ${ }^{1}$ and values derived from the critical compilation of Wiese et al. ${ }^{9}$ The level numbers correspond to those in Ref. 1. The errors given are the standard deviations evaluated from five independent runs.

For most of the levels, our results are in excellent agreement with the results of Adams and Whaling. ${ }^{1}$ We are in disagreement for the $3169.67-\AA$ lines of level 3 .

In our spectra, the intensity of this line is obviously smaller than that of the $3139.01-\AA$ line. This tendency is in agreement with the data presented by Wiese et al. ${ }^{9}$

The two lines originating from level 4 are slightly disturbed by blends on our spectra. We do not recommend their use for intensity calibration.

In our experiment, the branching ratio for level 5 is strongly dependent on the discharge conditions. For this reason, we have omitted level 5 in Table 1.

Concerning level 7, we have no explanation for the discrepancy, but we note that our mutual error bars still overlap. 
Table 1. Branching Ratios for Families of Lines from Upper Levels in Ar II ${ }^{a}$

\begin{tabular}{|c|c|c|c|c|c|c|}
\hline \multirow{2}{*}{\multicolumn{2}{|c|}{ Upper Level }} & \multirow[b]{3}{*}{$\lambda(\AA)$} & \multicolumn{4}{|c|}{$\mathrm{A}_{i j} / \mathrm{A}_{i 0}(\%)$} \\
\hline & & & \multirow{2}{*}{\multicolumn{2}{|c|}{$\begin{array}{c}\text { This } \\
\text { Experiment }\end{array}$}} & \multirow[b]{2}{*}{ CIT } & \multirow[b]{2}{*}{ NBS } \\
\hline No. & Designation & & & & & \\
\hline \multirow[t]{4}{*}{1.} & $4 p^{\prime 2} \mathrm{P}_{1 / 2}^{0}$ & 2891.61 & 0.214 & \pm 6 & 0.21 & - \\
\hline & & 2979.05 & 0.48 & \pm 2 & 0.48 & - \\
\hline & & 4131.73 & 1 & & 1 & - \\
\hline & & 4474.76 & 0.325 & \pm 2 & 0.323 & - \\
\hline \multirow[t]{7}{*}{2.} & $4 p^{\prime 2} \mathrm{P}_{3 / 2}^{\circ}$ & 2942.89 & 0.65 & \pm 2 & 0.67 & -. \\
\hline & & 3033.51 & 0.124 & \pm 2 & 0.126 & - \\
\hline & & 3766.12 & 0,096 & \pm 7 & 0.099 & - \\
\hline & & 4237.22 & 0.137 & \pm 2 & 0.139 & 0.21 \\
\hline & & 4277.52 & 1 & & 1 & 1 \\
\hline & & 4530.55 & 0.0269 & $9 \pm 4$ & 0.0251 & - \\
\hline & & 4598.76 & 0.083 & \pm 2 & 0.082 & - \\
\hline \multirow[t]{3}{*}{3.} & $4 d^{4} \mathrm{P}_{5 / 2}$ & 3139.01 & 0.37 & \pm 2 & 0.39 & 0.53 \\
\hline & & 3169.67 & 0.33 & \pm 2 & 0.43 & 0.43 \\
\hline & & 3868.52 & 1 & & 1 & 1 \\
\hline \multirow[t]{2}{*}{4.} & $4 d^{4} \mathrm{P}_{3 / 2}$ & 3249.80 & 0.70 & \pm 7 & 0.77 & 0.91 \\
\hline & & 3932.55 & 1 & & 1 & 1 \\
\hline \multirow[t]{2}{*}{6.} & $4 d^{4} \mathrm{D}_{7 / 2}$ & 3491.54 & 3.0 & \pm 2 & 2.9 & 3.2 \\
\hline & & 3780.84 & 1 & & 1 & 1 \\
\hline \multirow[t]{2}{*}{7.} & $4 p^{4} \mathrm{D}_{7 / 2}^{\circ}$ & 4348.06 & 7.4 & \pm 5 & 6.8 & - \\
\hline & & 6643.72 & 1 & & 1 & \\
\hline \multirow[t]{6}{*}{8.} & $4 p^{4} \mathrm{P}_{3 / 2}^{\circ}$ & 4371.33 & 0.393 & \pm 2 & 0.388 & 0.40 \\
\hline & & 4400.10 & 0.290 & \pm 1 & 0.288 & 0.28 \\
\hline & & 4420.91 & 0.054 & \pm 3 & 0.057 & 0.057 \\
\hline & & 4735.91 & 1 & & 1 & 1 \\
\hline & & 4933.21 & 0.249 & \pm 2 & 0.243 & 0.25 \\
\hline & & 5062.04 & 0.383 & \pm 1 & 0.381 & 0.38 \\
\hline \multirow[t]{3}{*}{9.} & $4 p^{\prime 2} \mathrm{~F}_{5 / 2}^{\circ}$ & 4637.23 & 0.342 & \pm 2 & 0.335 & 0.39 \\
\hline & & 5017.16 & 1 & & 1 & 1 \\
\hline & & 6172.29 & 0.93 & \pm 7 & 0.92 & - \\
\hline \multirow[t]{4}{*}{10.} & $4 p^{\prime 2} \mathbf{F}_{7 / 2}^{\circ}$ & 4609.56 & 1 & & 1 & 1 \\
\hline & & 4904.75 & 0.042 & \pm 2 & 0.044 & 0.049 \\
\hline & & 5141.79 & 0.098 & \pm 3 & 0.099 & 0.104 \\
\hline & & 6114.93 & 0.245 & \pm 6 & 0.235 & - \\
\hline
\end{tabular}

$a$ Values in the column headed CIT are from Adams and Whaling ${ }^{1}$; values in the column headed NBS are from Wiese et al. ${ }^{9}$

It can be concluded that for most of our data we find excellent agreement with Adams and Whaling, ${ }^{1}$ in that the scatter is smaller than $2 \%$.

The branching ratios for levels $1,2,6,8,9$, and 10 may be considered as reliable and thus suitable for intensity calibration purposes. That the results of this experiment were obtained under widely varying discharge conditions adds to the reliability of these branching ratios.

However, the deviations found for the levels $3-5$, and 7 indicate that, in spite of the obvious advantages and the huge amount of information provided by Fourier spectroscopy, there may still be undetected sources of systematic errors.

We are indebted to J. W. Brault for his continuing interest and generous cooperation. We would also like to thank Kitt Peak National Observatory for providing their facilities and the Deutsche Forschungsgemeinschaft for financial support. We gratefully acknowledge fruitful discussions with $\mathrm{E}$. Schulz-Gulde.

* Present address, Department of Physics, Stanford University, Stanford, California 94305.

\section{REFERENCES}

1. D. L. Adams and W. Whaling, "Argon branching ratios for spectral-intensity calibration," J. Opt. Soc. Am. 71, 1036-1038 (1981).

2. J. W. Brault, "Solar Fourier transform spectroscopy," in Proceedings of the JOSO workshop, Osservazioni e Memorie dell" Osservatorio Astrofisico di Arcetri, Firenze, Fascicolo 106, 33-52 (1978).

3. K. Danzmann and M. Kock, "Oscillator strengths of Ti II from combined hook and emission measurements," J. Phys. B 13, 2051-2059 (1980)

4. D. L. Adams and W. Whaling, "Level life times and branching ratios of the $z^{3} P^{\circ}$ term of neutral iron," J. Quant. Spectrosc. Radiat. Transfer 25, 233-236 (1981).

5. D. E. Blackwell et al., "Precision measurement of relative oscillator strenghts-IV. Attainment of 0.5 percent accuracy. Fe I transitions from levels $a^{5} \mathrm{D}_{0-4}(0.00-0.12 \mathrm{eV})$," Mon. Not. R. Astron. Soc. 186, 633-650 (1979).

6. D. E. Blackwell, A. D. Petford, and M. J. Shallis "Precision measurement of relative oscillator strengths-VI. Measures of Fe I transitions from levels $a^{5} \mathrm{~F}_{1-5}(0.86-1.01 \mathrm{eV})$ with an accuracy of 0.5 percent," Mon. Not. R. Astron. Soc. 186, 657-668 (1979).

7. D. E. Blackwell et al., "Precision measurement of relative oscillator strengths-VIII. Measures of Fe I transitions from the levels $a^{3} \mathrm{~F}_{2-4}(1.49-1.61 \mathrm{eV})$ with an accuracy of 1 percent," Mon. Not. R. Astron. Soc. 191, 445-450 (1980).

8. D. E. Blackwell et al., "Precision measurement of relative oscillator strengths-X. Measures of $\mathrm{Fe}$ I transitions from levels $a^{5} P_{1-3}$ $(2.18-2.28 \mathrm{eV}), a^{3} \mathrm{P}_{2}(2.28 \mathrm{eV}), a^{3} \mathrm{P}_{0,1}(2.49-2.42 \mathrm{eV}), z^{7} \mathrm{D}_{1-5}{ }^{0}$ $(2.48-2.40 \mathrm{eV})$, and $a^{3} \mathrm{H}_{4-6}(2.45-2.40 \mathrm{eV})$," Mon. No. R. Astron. Soc. (to be published).

9. W. L. Wiese, M. W. Smith, and B. M. Miles, "Atomic transition probabilities,” Nat. Stand. Ref. Data Ser., Nat. Bur. Stand. II (22), 187-210 (1969). 